\title{
On modular transformations of toric conformal blocks
}

\section{N. Nemkov}

Moscow Institute of Physics and Technology (MIPT),

Dolgoprudny, Russia

Institute for Theoretical and Experimental Physics (ITEP),

Moscow, Russia

E-mail: nnemkov@gmail.com

ABSTRACT: We derive and solve the difference equations on the toric modular kernel following from the consistency relations in the fusion algebra. The result is explicit and simple series expansion for the toric modular kernel of non-degenerate Virasoro conformal blocks. We show that this expansion is equivalent to the celebrated integral representation due to J. Teschner.

Keywords: Conformal and W Symmetry, Nonperturbative Effects, Quantum Groups

ARXIV EPRINT: 1504.04360 


\section{Contents}

1 Introduction 1

2 Conformal blocks $\quad 2$

2.1 General discussion 2

2.2 With degenerate fields 4

3 Fusion and modular transformations 5

3.1 General discussion 5

$\begin{array}{ll}3.2 & \text { With degenerate fields }\end{array}$

$\begin{array}{lll}4 & \text { Difference equations on the modular kernel } & 7\end{array}$

5 Modular kernel series expansion from the difference equations 13

$6 \quad$ Integral representation of the toric modular kernel 17

$\begin{array}{llr}7 \text { Conclusion } & 19\end{array}$

$\begin{array}{lr}\text { A Special functions } & 20\end{array}$

B Identity relating double and single expansions of the modular kernel 21

$\begin{array}{ll}\text { C Verifying difference equation with shift in } \mu & 23\end{array}$

\section{Introduction}

Conformal blocks are important ingredients for a wide variety of physical theories. Though being fully determined by the conformal symmetry they are not completely understood. A number of representations allowing for control over some of their properties, together with a limited amount of exactly solvable examples exists. In this paper we study one particular property of the conformal blocks, namely, their behavior under the modular transformations. This direction seems especially interesting for the following reason. First, usual definition of the conformal blocks keeps their properties under the modular transformations well hidden. Second, modular transformations can be studied indirectly, without calling for the explicit shapes of conformal blocks. Therefore, by investigating the modular transformations of conformal blocks we can hope to gain a deeper insight at the aspects of their structure which are not at all visible from the definition or conventional representations.

The modular transformations for generic conformal blocks must satisfy some polynomial relations the most famous of which is probably the pentagon identity. As turns out, 
specifying the shape of the fusion matrices for degenerate representations and imposing the fusion rules is enough to convert the pentagon identity into a set of difference equations uniquely determining generic fusion matrix as the solution with certain properties. Toric modular kernel enters similar polynomial relation which can also be used to derive a set of difference equations. As before, these equations define the toric modular kernel. In the paper we construct the solution to these equations in the form of a series expansion and show that this representation is equivalent to the well known integral formula.

\section{Conformal blocks}

\subsection{General discussion}

Conformal blocks are prime constituents of any conformal field theory (CFT) [1]. In the present paper we only deal with two-dimensional CFT. Symmetries of 2d CFT are encoded in the Virasoro algebra spanned by generators $L_{n}, n \in \mathbb{Z}$ with commutation relations

$$
\left[L_{n}, L_{m}\right]=(n-m) L_{n+m}+\frac{c}{12} n\left(n^{2}-1\right) \delta_{n+m, 0}
$$

In fact, the full symmetry algebra of the theory contains two copies of the Virasoro algebra (holomorphic + antiholomorphic). In what follows we will pretend that all operators in the theory are holomorphic. This will have no effect on our conclusions about conformal blocks which are by definition holomorphic objects. However, disregarding antiholomorphic part will greatly lighten the notation.

Space of states in CFT is isomorphic to the space of local operators. It is decomposed into the direct sum of the irreducible highest weight representations called Verma modules or conformal families. Highest weight vectors $|\Delta\rangle$ are eigenfunctions of $L_{0}$ and they are annihilated by all $L_{n}$ with positive $n$

$$
\begin{aligned}
& L_{0}|\Delta\rangle=\Delta \\
& L_{n}|\Delta\rangle=0, \quad n>0
\end{aligned}
$$

Verma module is conveniently organized into levels labeled by a non-negative integer $k$. Vectors of the form

$$
L_{-Y}|\Delta\rangle \equiv L_{-1}^{k_{1}} L_{-2}^{k_{2}} \ldots|\Delta\rangle
$$

constitute a basis at level $k$. Here $Y=\left\{k_{1}, k_{2}, \ldots\right\}$ is a partition of $k$, i.e. $|Y|=k_{1}+2 k_{2}+$ $\cdots=k$. Thus, the operator product expansion (OPE) in 2d CFT can be written in the following form

$$
\phi_{\Delta_{1}}(x) \phi_{\Delta_{2}}(0)=\sum_{\Delta, Y} C_{\Delta_{1} \Delta_{2}}^{\Delta, Y}(x) L_{-Y} \phi_{\Delta}(0)
$$

Conformal invariance fixes functions $C_{\Delta_{1} \Delta_{2}}^{\Delta, Y}(x)$ up to constants $C_{\Delta_{1} \Delta_{2}}^{\Delta}$ independent of partition $Y$

$$
C_{\Delta_{1} \Delta_{2}}^{\Delta, Y}(x)=C_{\Delta_{1} \Delta_{2}}^{\Delta} \beta_{\Delta_{1} \Delta_{2}}^{\Delta, Y} x^{\Delta-\Delta_{1}-\Delta_{2}}
$$


One normalizes by definition $\beta_{\Delta_{1} \Delta_{2}}^{\Delta, \varnothing}=1$, then $C_{\Delta_{1} \Delta_{2}}^{\Delta}$ is a three-point correlation function

$$
C_{\Delta_{1}, \Delta_{2}}^{\Delta_{3}}=\left\langle\phi_{\Delta_{1}}(0) \phi_{\Delta_{2}}(1) \phi_{\Delta_{3}}(\infty)\right\rangle
$$

Here a field inserted at infinity is understood as limit $\phi_{\Delta}(\infty)=\lim _{z \rightarrow \infty} z^{2 \Delta} \phi_{\Delta}(z)$. One should stress that coefficients $\beta_{\Delta_{1} \Delta_{2}}^{\Delta, Y}$ are completely fixed by the conformal symmetry (requirement that both sides in (2.4) transform identically).

By means of the OPE one can decompose any correlation function into a combination of conformal blocks. Consider the four-point correlation function on a sphere ${ }^{1}$

$$
\langle\underbrace{\phi_{\Delta_{1}}(0) \phi_{\Delta_{2}}(x)}_{\mathrm{OPE}} \underbrace{\phi_{\Delta_{3}}(1) \phi_{\Delta_{4}}(\infty)}_{O P E}\rangle=\sum_{\Delta} C_{\Delta_{1} \Delta_{2}}^{\Delta} C_{\Delta_{3} \Delta_{4}}^{\Delta} B_{\Delta}\left[\begin{array}{cc}
\Delta_{2} \Delta_{3} \\
\Delta_{1} \Delta_{4}
\end{array}\right](x)
$$

Here function $B_{\Delta}\left[\Delta_{i}\right](x)$ is the spheric conformal block. It is conveniently depicted graphically as

$$
B_{\Delta}\left[\begin{array}{ll}
\Delta_{2} & \Delta_{3} \\
\Delta_{1} & \Delta_{4}
\end{array}\right](x)=\underbrace{}_{\Delta_{1}} \Delta \Delta_{4}
$$

By definition, conformal block is a series in powers of $x$

$$
B_{\Delta}\left[\begin{array}{cc}
\Delta_{2} & \Delta_{3} \\
\Delta_{1} & \Delta_{4}
\end{array}\right](x)=x^{\Delta-\Delta_{1}-\Delta_{2}} \sum_{n=0}^{\infty} x^{n} B_{\Delta}^{(n)}\left[\begin{array}{cc}
\Delta_{2} & \Delta_{3} \\
\Delta_{1} & \Delta_{4}
\end{array}\right]
$$

where

$$
B_{\Delta}^{(n)}\left[\begin{array}{cc}
\Delta_{2} & \Delta_{3} \\
\Delta_{1} & \Delta_{4}
\end{array}\right]=\sum_{\substack{Y, Y^{\prime} \\
|Y|=\left|Y^{\prime}\right|=n}} \beta_{\Delta_{1} \Delta_{2}}^{\Delta, Y} \beta_{\Delta_{1} \Delta_{2}}^{\Delta, Y}\left\langle L_{-Y} \phi(0) L_{-Y^{\prime}} \phi(\infty)\right\rangle
$$

We emphasize that conformal block (2.9) is unambiguously fixed by conformal symmetry. We present here first few terms of the $x$-expansion

$$
\begin{aligned}
B_{\Delta} & {\left[\begin{array}{ll}
\Delta_{2} & \Delta_{3} \\
\Delta_{1} & \Delta_{4}
\end{array}\right](x)=x^{\Delta-\Delta_{1}-\Delta_{2}}\left(1+x \frac{\left(\Delta-\Delta_{1}+\Delta_{2}\right)\left(\Delta+\Delta_{3}-\Delta_{4}\right)}{2 \Delta}\right.} \\
& \left.+x^{2}\left(\frac{(c+8 \Delta)\left(\Delta-\Delta_{1}+\Delta_{2}\right)\left(1+\Delta-\Delta_{1}+\Delta_{2}\right)\left(\Delta+\Delta_{3}-\Delta_{4}\right)\left(1+\Delta+\Delta_{3}-\Delta_{4}\right)}{4 \Delta\left(c-10 \Delta+2 c \Delta+16 \Delta^{2}\right)}+\ldots\right)+O\left(x^{3}\right)\right)
\end{aligned}
$$

Already the second-order coefficient here is bulky and we only show it partially masking the remainder by ellipses.

We now turn to the CFT on a torus. Simplest non-trivial example of the toric blocks is the conformal block for the one-point correlation function

$$
B_{\Delta}\left(\Delta_{\text {ext }} \mid \tau\right)=\operatorname{Tr}_{\Delta}\left(q^{L_{0}-c / 24} \phi_{\Delta_{\text {ext }}}(0)\right), \quad q=e^{2 \pi i \tau}
$$

\footnotetext{
${ }^{1}$ We want to stress that correlation functions are in fact bilinear combinations of holomorphic and antiholomorphic conformal blocks. Thus formula (2.7) does not hold literally.
} 
or, graphically

$$
B_{\Delta}\left(\Delta_{\text {ext }} \mid \tau\right)=\Delta_{\text {ext }}
$$

Here $\tau$ is the modular parameter of the torus. The trace is taken over the Verma module of primary dimension $\Delta ; \Delta_{\text {ext }}$ stays for the dimension of the external field. Similarly to the spheric case the toric conformal block naturally admits expansion in powers of $q$

$$
\begin{aligned}
B_{\Delta}\left(\Delta_{\text {ext }} \mid \tau\right)= & q^{\Delta-c / 24} \sum_{n=0}^{\infty} q^{n} B_{\Delta}^{(n)}\left(\Delta_{\text {ext }}\right) \\
=q^{\Delta-c / 24}(1+ & q\left(\frac{\Delta_{\text {ext }}\left(\Delta_{\text {ext }}-1\right)}{2 \Delta}+1\right) \\
& \left.\quad+q^{2}\left(\frac{(8 \Delta+c) \Delta_{\text {ext }}^{4}}{4 \Delta\left(c+2 c \Delta-10 \Delta+16 \Delta^{2}\right)}+\ldots\right)+O\left(q^{3}\right)\right)
\end{aligned}
$$

For the sake of brevity part of the second-order coefficient is hidden in ellipses.

Apart from certain special cases conformal blocks are best understood as such $x$ - or $q$-series expansions. There are, however, a few exceptions when conformal blocks can be found in closed forms. Those of the most interest for the present paper are discussed in the next subsection.

\section{$2.2 \quad$ With degenerate fields}

Conformal blocks can be found in a closed form when one of the external dimensions takes a specific value corresponding to a degenerate representation of the Virasoro algebra. To describe these efficiently we introduce the Liouville-type parametrization for the central charge and conformal dimensions

$$
c=1+6 Q^{2}, \quad Q=b+b^{-1}, \quad \Delta(a)=a(Q-a)
$$

Later in the article we will use $\alpha$-variables for some momentums instead of $a$. The relation is

$$
a=\alpha+Q / 2, \quad \Delta(\alpha)=\frac{Q^{2}}{4}-\alpha^{2}
$$

Degenerate dimensions correspond to the following Liouville momentums

$$
\Delta_{\mathrm{deg}}=a_{\mathrm{deg}}\left(Q-a_{\mathrm{deg}}\right), \quad a_{\mathrm{deg}}=-\frac{n b}{2}-\frac{m b^{-1}}{2}, \quad n, m \geq 0
$$

When conformal dimension of a field is degenerate its Verma module contains a singular vector. As a consequence, correlation functions and conformal blocks involving a degenerate field satisfy certain differential equations.

We illustrate this at the simplest and most important for our purposes case of the degenerate field with the Liouville momentum $-b / 2$. From commutation relations of the Virasoro algebra (2.1) it is easy to check that vector

$$
|\operatorname{sing}\rangle=\left(L_{-1}^{2}+b^{2} L_{-2}\right)|\Delta(-b / 2)\rangle
$$


is indeed a singular vector, i.e. it is annihilated by all $L_{n}$ with $n>0$ (this is only non-trivial for $n=1,2)$. In effect, any correlation function involving $|\operatorname{sing}\rangle$ must vanish and for the four-point correlation function

$$
C\left[\begin{array}{cc}
\Delta(-b / 2) & \Delta_{3} \\
\Delta_{1} & \Delta_{4}
\end{array}\right](x)=\left\langle\phi_{\Delta_{1}}(0) \phi_{\Delta(-b / 2)}(x) \phi_{\Delta_{3}}(1) \phi_{\Delta_{4}}(\infty)\right\rangle
$$

presence of the singular state produces equation

$$
\left(b^{-2} x(1-x) \partial_{x}^{2}+(2 x-1) \partial_{x}+\Delta(-b / 2)+\frac{\Delta_{1}}{x}-\frac{\Delta_{3}}{x-1}-\Delta_{4}\right) C\left[\begin{array}{cc}
\Delta(-b / 2) & \Delta_{3} \\
\Delta_{1} & \Delta_{4}
\end{array}\right](x)=0
$$

Two independent solutions to this equation can be chosen as

$$
\begin{aligned}
& B_{a_{1}-b / 2}\left[\begin{array}{cr}
-b / 2 & a_{3} \\
a_{1} & a_{4}
\end{array}\right](x)=x^{b a_{1}}(1-x)^{b a_{3}}{ }_{2} F_{1}(A, B ; C \mid x) \\
& B_{a_{1}+b / 2}\left[\begin{array}{cr}
-b / 2 & a_{3} \\
a_{1} & a_{4}
\end{array}\right](x)=x^{b\left(Q-a_{1}\right)}(1-x)^{b a_{3}}{ }_{2} F_{1}(1+A-C, 1+B-C ; 2-C \mid x)
\end{aligned}
$$

where

$$
A=-\frac{b^{2}}{2}+b\left(a_{1}+a_{3}-a_{4}\right), \quad B=b\left(a_{1}+a_{3}+a_{4}\right)+2 \Delta(-b / 2), \quad C=-b^{2}+2 b a_{1}
$$

One can verify in $x$-expansion that expressions in the r.h.s. of (2.21) coincide with the general formula for the $x$-expansion of conformal blocks (which is partially presented in equation (2.11)) upon substituting to the latter parametrization (2.15) and specifying $a=$ $a_{1} \pm b / 2, a_{2}=-b / 2$.

Thus, for correlation function (2.19) only two conformal blocks (2.21) in decomposition (2.7) are relevant. This is a manifestation of the fusion rules for the OPE involving degenerate fields. Particularly

$$
\phi_{a} \times \phi_{-b / 2}=\phi_{a+b / 2}+\phi_{a-b / 2}
$$

In words, only operators of momentums $a \pm b / 2$ may have non-vanishing coefficients in the OPE of fields $\phi_{a}, \phi_{-b / 2}$. Hence the space of conformal blocks is two-dimensional and functions (2.21) can be chosen as a basis.

\section{$3 \quad$ Fusion and modular transformations}

\subsection{General discussion}

In order to obtain decomposition (2.7) we have chosen a particular pairing of the fields in the correlation function. Namely, we fused the fields with dimensions $\Delta_{1}, \Delta_{2}$ and $\Delta_{3}, \Delta_{4}$. Fusing the fields in a different way would result in a different basis for conformal blocks. For example

$$
\langle\underbrace{\phi_{\Delta_{1}}(0) \phi_{\Delta_{4}}(\infty)}_{\mathrm{OPE}} \underbrace{\phi_{\Delta_{2}}(x) \phi_{\Delta_{3}}(1)}_{O P E}\rangle=\sum_{\Delta} C_{\Delta_{1} \Delta_{4}}^{\Delta} C_{\Delta_{2} \Delta_{3}}^{\Delta} B_{\Delta}^{t}\left[\begin{array}{cc}
\Delta_{2} \Delta_{3} \\
\Delta_{1} & \Delta_{4}
\end{array}\right](x)
$$


Function $B_{\Delta}^{t}\left[\Delta_{i}\right](x)$ appearing in this decomposition is called the t-channel conformal block (in contranst to (2.8) which is called the s-channel conformal block) and depicted as

$$
B_{\Delta}^{t}\left[\begin{array}{ll}
\Delta_{2} & \Delta_{3} \\
\Delta_{1} & \Delta_{4}
\end{array}\right](x)=\bar{\Delta}_{\Delta_{1}}^{\Delta_{2}} \Delta_{4}^{\Delta_{3}}
$$

Throughout this section we introduce additional labels $s$ and $t$ to differentiate between the s- and t-channel blocks. In the subsequent sections we only use the s-channel blocks and hence, drop the superscript. There is a simple relation between the s- and t- channel conformal blocks

$$
B_{\Delta}^{t}\left[\begin{array}{ll}
\Delta_{2} & \Delta_{3} \\
\Delta_{1} & \Delta_{4}
\end{array}\right](x)=B_{\Delta}^{s}\left[\begin{array}{cc}
\Delta_{2} & \Delta_{1} \\
\Delta_{3} & \Delta_{4}
\end{array}\right](1-x)
$$

From asymptotic near $x=0(2.9)$ one sees that the s-channel conformal blocks are linearly independent. Then, from (3.3) we conclude that the t-channel conformal blocks are also linearly independent. Therefore, decompositions into the s- and t-channels (2.7), (3.1) are simply decompositions in different bases. Hence these bases are related by some transformation matrix

$$
B_{\Delta}^{s}\left[\begin{array}{cc}
\Delta_{2} & \Delta_{3} \\
\Delta_{1} & \Delta_{4}
\end{array}\right](x)=\sum_{\Delta^{\prime}} F_{\Delta \Delta^{\prime}}\left[\begin{array}{cc}
\Delta_{2} & \Delta_{3} \\
\Delta_{1} & \Delta_{4}
\end{array}\right] B_{\Delta}^{t}\left[\begin{array}{cc}
\Delta_{2} & \Delta_{3} \\
\Delta_{1} & \Delta_{4}
\end{array}\right](x)
$$

Here the summation is performed over the spectrum of primary fields' dimensions. One might question formula (3.4) when the spectrum is continuous, but its validity is a common belief confirmed from various perspectives. Transformation from the t- to the s-channel is sometimes called fusion. Hence, we call matrix $F_{\Delta \Delta^{\prime}}\left[\Delta_{i}\right]$ the fusion kernel.

From relation between the s- and t-channel (3.3) we see that the fusion kernel not only describes fusion transformation, but rather monodromy properties of the s-channel conformal block itself

$$
B_{\Delta}^{s}\left[\begin{array}{ll}
\Delta_{2} & \Delta_{3} \\
\Delta_{1} & \Delta_{4}
\end{array}\right](x)=\sum_{\Delta^{\prime}} F_{\Delta \Delta^{\prime}}\left[\begin{array}{cc}
\Delta_{2} & \Delta_{3} \\
\Delta_{1} & \Delta_{4}
\end{array}\right] B_{\Delta}^{s}\left[\begin{array}{cc}
\Delta_{2} & \Delta_{1} \\
\Delta_{3} & \Delta_{4}
\end{array}\right](1-x)
$$

and therefore contains non-trivial information about the structure of the conformal blocks.

Correlation functions on a torus must be invariant under the action of $\operatorname{SL}(2, \mathbb{Z})$ generated by $T: \tau \rightarrow \tau+1$ and $S: \tau \rightarrow-1 / \tau$. As a consequence, toric conformal blocks form a representation of the modular group. $T$-transformation acts diagonally and can be read off directly from definition $(2.12)$

$$
B_{\Delta}\left(\Delta_{\text {ext }} \mid \tau+1\right)=q^{\Delta-c / 24} B_{\Delta}(\tau)
$$

The action of $S$ is non-trivial and close in spirit to the fusion transformation (3.4)

$$
B_{\Delta}\left(\Delta_{\text {ext }} \mid-1 / \tau\right)=\sum_{\Delta^{\prime}} M_{\Delta \Delta^{\prime}}\left(\Delta_{\text {ext }}\right) B_{\Delta^{\prime}}\left(\Delta_{\text {ext }} \mid \tau\right)
$$

We call matrix $M_{\Delta \Delta^{\prime}}$ the toric modular kernel or simply the modular kernel. 
The present paper is mainly concerned with the toric modular kernel. It is simpler then the fusion kernel (already by counting the number of parameters) and allows for a clearer exposition while catching all the important features. In paper [2] an integral expression describing modular kernel for generic conformal blocks was proposed. We postpone discussion of this formula until section (6).

In the following, for the sake of brevity we often use the term modular transformations for both, fusion transformations of the spheric and S-transformations of the toric blocks. Hopefully, this is not to be a source of confusion.

\subsection{With degenerate fields}

In practice it turns out to be more convenient to use the Liouville-type parametrization (2.15). Hence we rewrite (3.5) as

$$
B_{a}\left[\begin{array}{ll}
a_{2} & a_{3} \\
a_{1} & a_{4}
\end{array}\right](x)=\int d a^{\prime} F_{a a^{\prime}}\left[\begin{array}{ll}
a_{2} & a_{3} \\
a_{1} & a_{4}
\end{array}\right] B_{a^{\prime}}\left[\begin{array}{ll}
a_{2} & a_{1} \\
a_{3} & a_{4}
\end{array}\right](1-x)
$$

Note also that we have dropped the s-channel superscript. Here and in the sequel the spheric conformal block without a superscript always refers to the s-channel conformal block.

Consider conformal blocks with a degenerate field of momentum $-b / 2$ (2.21). Recall that due to the fusion rules (2.23) the space of conformal blocks is two-dimensional. Basis s-channel conformal blocks are given by formulas (2.21). Let us introduce notation

$$
B_{ \pm}^{s}=B_{a_{1} \pm b / 2}^{s}\left[\begin{array}{cc}
-b / 2 & a_{3} \\
a_{1} & a_{4}
\end{array}\right](x)
$$

Note that permutation $a_{1} \leftrightarrow a_{3}$ acts on parameters $A, B, C(2.22)$ in the following way

$$
A \rightarrow A, \quad B \rightarrow B, \quad C \rightarrow A+B-C+1
$$

Therefore, in this case the fusion kernel is nothing else than the matrix of connection coefficients for the hypergeometric function

$$
\left(\begin{array}{l}
B_{+}^{s} \\
B_{-}^{s}
\end{array}\right)=F\left(\begin{array}{c}
B_{+}^{t} \\
B_{-}^{t}
\end{array}\right), \quad F\left[\begin{array}{cc}
-b / 2 & a_{3} \\
a_{1} & a_{4}
\end{array}\right]=\left(\begin{array}{cc}
\frac{\Gamma(2-C) \Gamma(A+B-C)}{\Gamma(1+A-C) \Gamma(1+B-C)} & \frac{\Gamma(2-C) \Gamma(-A-B+C)}{\Gamma(1-A) \Gamma(1-B)} \\
\frac{\Gamma(A+B-C) \Gamma(C)}{\Gamma(A) \Gamma(B)} & \frac{\Gamma(C) \Gamma(-A-B+C)}{\Gamma(-A+C) \Gamma(-B+C)}
\end{array}\right)
$$

\section{Difference equations on the modular kernel}

In this section we derive a set of the difference equations on the modular kernel (see (4.24), (4.25), (4.26)). These equations follow from the relations in the fusion/modular algebra. We first illustrate the idea at the example of the celebrated pentagon identity.

As is well known the fusion kernel satisfies the pentagon identity

$$
F_{p_{1} q_{2}}\left[\begin{array}{ll}
a_{2} & a_{3} \\
a_{1} & p_{2}
\end{array}\right] F_{p_{2} q_{1}}\left[\begin{array}{ll}
q_{2} & a_{4} \\
a_{1} & a_{5}
\end{array}\right]=\sum_{l} F_{p_{2} l}\left[\begin{array}{ll}
a_{3} & a_{4} \\
p_{1} & a_{5}
\end{array}\right] F_{p_{1} q_{1}}\left[\begin{array}{cc}
a_{2} & l \\
a_{1} & a_{5}
\end{array}\right] F_{l q_{2}}\left[\begin{array}{ll}
a_{2} & a_{3} \\
q_{1} & a_{4}
\end{array}\right]
$$


This formula follows from the requirement of consistency for the fusion transformations of the 5-point conformal blocks. Labels $a_{1-5}$ indicate the external momentums in these 5point blocks. When one of these momentums, say $a_{2}$, is set to a degenerate value $a_{2}=-b / 2$ two interesting phenomenons happen:

1) Three out of five fusion matrices entering the pentagon identity become degenerate and therefore known explicitly. The two other matrices are left with generic values of parameters. This converts polynomial equation (4.1) into a linear equation on the generic fusion matrices with the degenerate fusion matrices playing the role of coefficients.

2) Due to the fusion rules (2.23) generally continuous range of summation over momentum $l$ is restricted to just two values $l=q_{1} \pm b / 2$.

This turns the pentagon identity into a second order linear difference equation. One can attempt to solve this equation directly. In the previous work [3] we took this route and provided a way to recursively find coefficients in the expansion of the type (5.14) for the fusion kernel. In the current work we continue to develop this approach at the example of the toric modular kernel. This case appears to be simpler and allows for more complete understanding.

Now we proceed to the derivation of equations (4.24), (4.25), (4.26), which follow from the genus-one counterpart of the pentagon identity. Recall that in order to obtain the pentagon identity one needs to consider the transformation properties of the five-point conformal blocks. Likewise, in order to derive equations on the toric modular kernel we have to consider not a one-point toric conformal block, but a two-point block

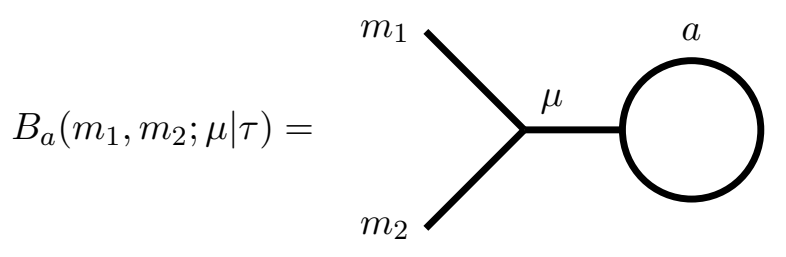

Operators corresponding to the legs $m_{1}, m_{2}$ are inserted close to each other somewhere on the torus. Conformal block also depends on their relative position, but we will not denote this dependence in a manifest way.

When one of the legs, say $m_{2}$, travels a closed path around the torus the conformal block acquires a monodromy. We can express this monodromy via the fusion matrices by representing the closed path at hand as a number of analytic continuations each performed by the corresponding operation on the four-point block. Subsequent manipulations are close to those used to obtain the Verlinde [4] formula and were extensively applied in papers $[5,6]$ to compute Wilson/'t Hooft loops in AGT dual gauge theories.

Assume that a closed loop in our pictorial representation of the two-point block is the $B$-cycle of the torus. Then, the series of moves that represents the transport of $m_{2}$ operator 
along the $A$ cycle is

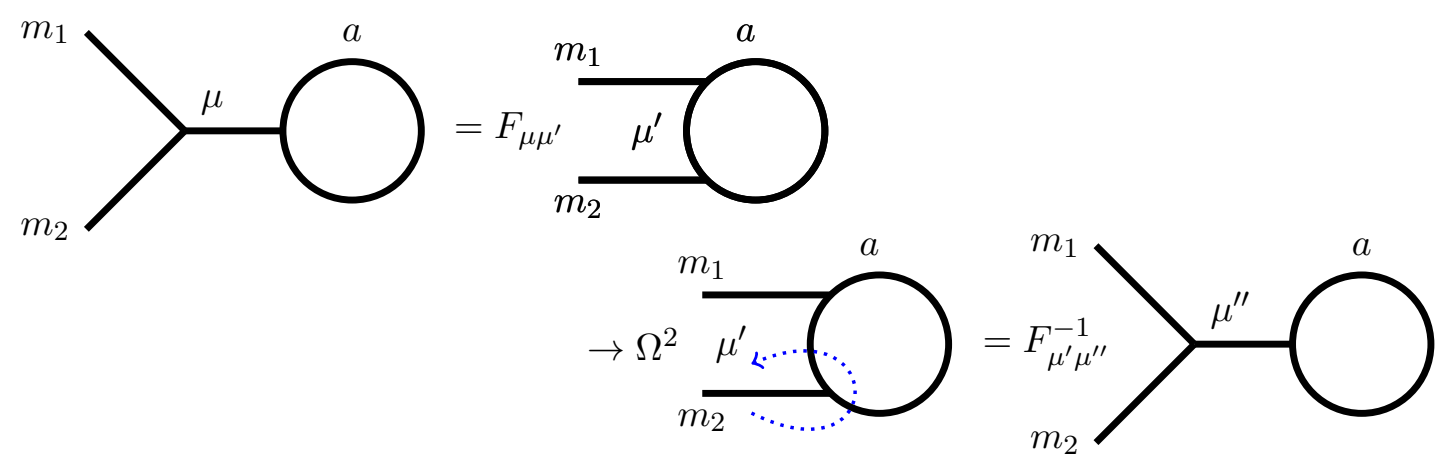

Therefore, we have the following expression for the conformal block continued along $A$-cycle

$$
A \circ B_{a}\left(m_{1}, m_{2} ; \mu \mid \tau\right)=\sum_{\mu^{\prime}, \mu^{\prime \prime}} F_{\mu \mu^{\prime}}\left[\begin{array}{cc}
m_{1} & a \\
m_{2} & a
\end{array}\right] \Omega^{2}\left(\mu^{\prime} ; m_{2}, a\right) F_{\mu^{\prime} \mu^{\prime \prime}}^{-1}\left[\begin{array}{cc}
m_{1} & a \\
m_{2} & a
\end{array}\right] B_{a}\left(m_{1}, m_{2} ; \mu^{\prime \prime} \mid \tau\right)
$$

In words: the first move uncouples two external legs; the second move transports the $m_{2}$ leg along the $A$-cycle (denoted by the dotted arrow); the third move fuses the two external operators back together. Quantities $F_{a a^{\prime}}$ are the same fusion matrices that relate the sand t-channel spheric conformal blocks while $\Omega\left(a_{1}, a_{2}, a_{3}\right)$ is the phase factor representing the monodromy of the permutation of the two legs in the conformal block which are 'close' to each other. It can be read off from the OPE asymptotic and equals simply

$$
\Omega\left(a_{1} ; a_{2}, a_{3}\right)=e^{i \pi\left(\Delta\left(a_{1}\right)-\Delta\left(a_{2}\right)-\Delta\left(a_{3}\right)\right)}
$$

Similarly, $B$-cycle monodromy is represented as

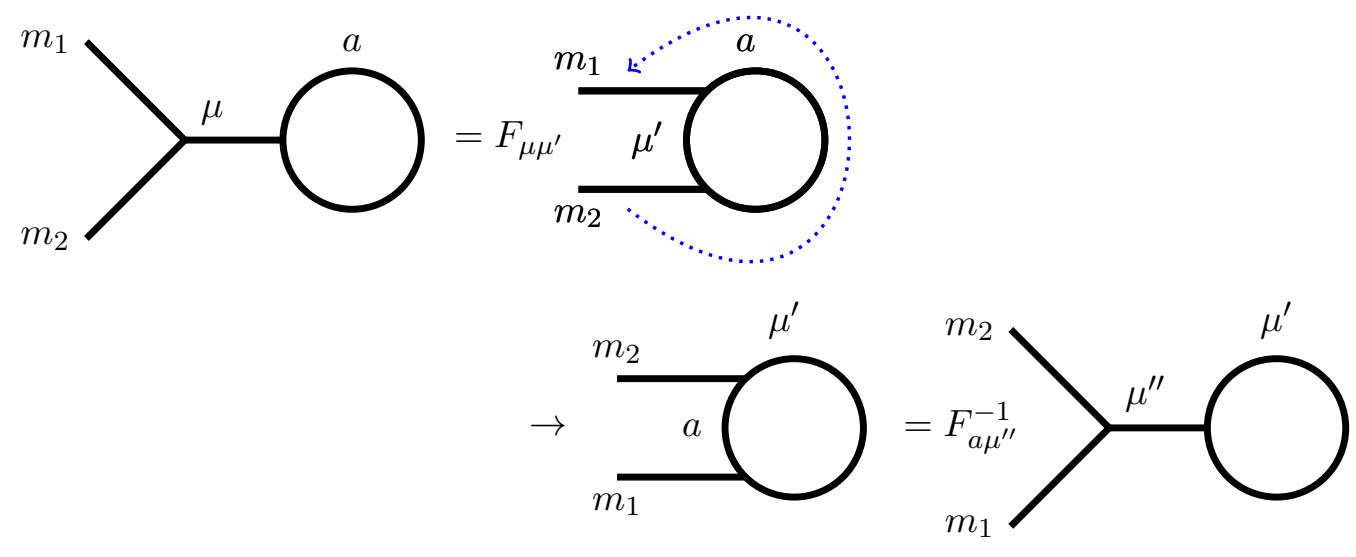

The first and the last moves here are the same as for the A-cycle. However, in contrast to the A-cycle transport along the direction of the B-cycle does not introduce a phase factor 
but simply permutes the two intermediate dimensions $a \leftrightarrow \mu^{\prime}$. Quantitatively

$$
B \circ B_{a}\left(m_{1}, m_{2} ; \mu \mid \tau\right)=\sum_{\mu^{\prime}, \mu^{\prime \prime}} F_{\mu \mu^{\prime}}\left[\begin{array}{ll}
m_{1} & a \\
m_{2} & a
\end{array}\right] F_{a \mu^{\prime \prime}}^{-1}\left[\begin{array}{ll}
m_{2} & \mu^{\prime} \\
m_{1} & \mu^{\prime}
\end{array}\right] B_{\mu^{\prime}}\left(m_{2}, m_{1} ; \mu^{\prime \prime} \mid \tau\right)
$$

Now, since the modular S-transformation permutes A and B cycles we have the following consistency condition

$$
S \circ A=B \circ S
$$

Spelled out explicitly it reads

$$
\begin{aligned}
M_{a a^{\prime}}(\mu) \sum_{\mu^{\prime \prime}} F_{\mu \mu^{\prime \prime}}\left[\begin{array}{ll}
m_{1} & a^{\prime} \\
m_{2} & a^{\prime}
\end{array}\right] \Omega^{2}\left(\mu^{\prime \prime} ; m_{2}, a^{\prime}\right) F_{\mu^{\prime \prime} \mu^{\prime}}^{-1}\left[\begin{array}{ll}
m_{1} & a^{\prime} \\
m_{2} & a^{\prime}
\end{array}\right]= \\
\sum_{a^{\prime \prime}} F_{\mu a^{\prime \prime}}\left[\begin{array}{ll}
m_{1} & a \\
m_{2} & a
\end{array}\right] F_{a \mu^{\prime}}^{-1}\left[\begin{array}{ll}
m_{2} & a^{\prime \prime} \\
m_{1} & a^{\prime \prime}
\end{array}\right] M_{a^{\prime \prime} a^{\prime}}\left(\mu^{\prime}\right)
\end{aligned}
$$

This is the analog of the pentagon identity intertwining spheric and toric transformation matrices.

We can turn this equation into a second-order difference equation along the lines described after equation (4.1). Namely, set momentum $m_{2}$ to a degenerate value $m_{2}=-b / 2$. Due to the fusion rules (2.23) for $m_{2}=-b / 2$ we have the following selection rules on the momentums entering equation (4.9)

$$
\mu=m_{1}+s_{1} b / 2, \quad \mu^{\prime \prime}=m_{1}+s_{2} b / 2, \quad \mu^{\prime}=a^{\prime}+s_{3} b / 2, \quad a^{\prime \prime}=a+s_{4} b / 2
$$

where all quantities $s_{1}, s_{2}, s_{3}, s_{4}$ are either + or - . Denote

$$
F_{a=a_{1}+s_{1} b / 2, a^{\prime}=a_{3}+s_{2} b / 2}\left[\begin{array}{cc}
-b / 2 & a_{3} \\
a_{1} & a_{4}
\end{array}\right]=F_{s_{1}, s_{2}}\left[\begin{array}{cc}
-b / 2 & a_{3} \\
a_{1} & a_{4}
\end{array}\right]
$$

Then, equation (4.9) for $m_{2}=-b / 2$ can be rewritten as

$$
\begin{aligned}
& M_{a a^{\prime}}(\mu) \sum_{s_{2}= \pm} F_{s_{1}, s_{2}}\left[\begin{array}{cr}
\mu-s_{1} b / 2 & a^{\prime} \\
-b / 2 & a^{\prime}
\end{array}\right] \Omega^{2}\left(a^{\prime}+s_{2} b / 2 ;-b / 2, a^{\prime}\right) F_{s_{2}, s_{3}}^{-1}\left[\begin{array}{cr}
\mu-s_{1} b / 2 & a^{\prime} \\
-b / 2 & a^{\prime}
\end{array}\right] \\
= & \sum_{s_{4}= \pm} F_{s_{1}, s_{4}}\left[\begin{array}{cr}
\mu-s_{1} b / 2 & a \\
-b / 2 & a
\end{array}\right] F_{-s_{4}, s_{3}}^{-1}\left[\begin{array}{cr}
b / 2 & a+s_{4} b / 2 \\
\mu-s_{1} b / 2 & a+s_{4} b / 2
\end{array}\right] M_{a+s_{4} b / 2, a^{\prime}}\left(\mu+\left(s_{3}-s_{1}\right) b / 2\right)
\end{aligned}
$$

This is indeed a difference equation on the modular kernel $M_{a a^{\prime}}(\mu)$. Note that for $s_{1}=s_{3}$ only shifts in the internal momentum $a$ are presented in the equation, while for $s_{1} \neq s_{3}$ we also have shifts in the external momentum $\mu$.

All of the fusion matrices entering equation (4.12) are known explicitly (3.11). Moreover, we can renormalize the basis of conformal blocks in a such a way that these matrices 
greatly simplify. Namely, we introduce the following renormalization factor for a single chiral vertex

$$
V\left(a_{1}, a_{2} ; a_{3}\right)=\frac{\Gamma_{b}\left(2 a_{1}\right) \Gamma_{b}\left(2 a_{2}\right) \Gamma_{b}\left(2 Q-2 a_{3}\right)}{\Gamma_{b}\left(2 Q-a_{1}-a_{2}-a_{3}\right) \Gamma_{b}\left(a_{1}+a_{2}-a_{3}\right) \Gamma_{b}\left(a_{1}-a_{2}+a_{3}\right) \Gamma_{b}\left(-a_{1}+a_{2}+a_{3}\right)}
$$

Here $\Gamma_{b}(z)$ is a special function we call the double gamma function (see appendix A). The four-point spheric conformal block contains two vertices and is renormalized as (in the following we use curly notation for the renormalized quantities)

$$
\mathcal{B}_{a}\left[\begin{array}{ll}
a_{2} & a_{3} \\
a_{1} & a_{4}
\end{array}\right](x)=V\left(a_{1}, a_{2} ; a\right) V\left(a, a_{3} ; a_{4}\right) B_{a}\left[\begin{array}{cc}
a_{2} & a_{3} \\
a_{1} & a_{4}
\end{array}\right](x)
$$

Toric conformal block contains a single vertex and thus is renormalized as

$$
\mathcal{B}_{a}(\mu \mid \tau)=V(\mu, a ; a) B_{a}(\mu \mid \tau)
$$

The fusion and the modular kernels are renormalized accordingly

$$
\mathcal{F}_{a a^{\prime}}\left[\begin{array}{ll}
a_{2} & a_{3} \\
a_{1} & a_{4}
\end{array}\right]=\frac{V\left(a_{1}, a_{2} ; a\right) V\left(a, a_{3} ; a_{4}\right)}{V\left(a_{3}, a_{2} ; a^{\prime}\right) V\left(a^{\prime}, a_{1} ; a_{4}\right)} F_{a a^{\prime}}\left[\begin{array}{cc}
a_{2} & a_{3} \\
a_{1} & a_{4}
\end{array}\right]
$$

and

$$
\mathcal{M}_{a a^{\prime}}(\mu)=\frac{V(\mu, a ; a)}{V\left(\mu, a^{\prime} ; a^{\prime}\right)} M_{a a^{\prime}}(\mu)
$$

Formula (4.16) holds for generic values of momentums. If we now set $a_{2}=-b / 2$ then, according to the fusion rules (2.23)

$$
a=a_{1}+s_{1} b / 2, \quad a^{\prime}=a_{3}+s_{2} b / 2
$$

with $s_{1}, s_{2}= \pm$. For this special choice of parameters the ratios of the double gamma functions in the renormalization factor (4.16) reduce to the ordinary gamma functions which in turn almost cancel with those in the standard normalization (3.11). The result is

$$
\mathcal{F}_{s_{1}, s_{2}}\left[\begin{array}{cr}
-b / 2 & a_{3} \\
a_{1} & a_{4}
\end{array}\right]=s_{1} \frac{\left.\sin \pi b\left(a_{4}+s_{1} a_{3}-s_{2} a_{1}-\left(1+s_{1}-s_{2}\right) b / 2\right)\right)}{\sin \pi b\left(2 a_{3}-b\right)}
$$

With this simple form of the degenerate fusion matrices it is easy to cast equations (4.12) explicitly. There are in fact four of them, corresponding to each choice of $s_{1}, s_{3}= \pm$. Equations for $s_{1}=s_{3}=1$ and $s_{1}=-s_{3}=1$ are

$$
\begin{gathered}
\left(\frac{\sin \pi b(2 a-b+\mu)}{\sin \pi b(2 a-b)} e^{\frac{b}{2} \partial_{a}}+\frac{\sin \pi b(2 a-b-\mu)}{\sin \pi b(2 a-b)} e^{-\frac{b}{2} \partial_{a}}\right) \mathcal{M}_{a a^{\prime}}(\mu)=-2 \cos \pi b\left(2 a^{\prime}-b\right) \mathcal{M}_{a a^{\prime}}(\mu) \\
\frac{1}{\sin \pi b(2 a-b)}\left(e^{\frac{b}{2} \partial_{a}}-e^{-\frac{b}{2} \partial_{a}}\right) \mathcal{M}_{a a^{\prime}}(\mu-b)=2 \mathcal{M}_{a a^{\prime}}(\mu)
\end{gathered}
$$


Equations with $s_{1}=s_{3}=-1$ and $s_{1}=-s_{3}=-1$ are equivalent to the above equations. Upon redefinition

$$
a=\alpha+Q / 2, \quad a^{\prime}=\alpha^{\prime}+Q / 2
$$

these equations become (4.24) and (4.26). Equation (4.25) can be derived from condition

$$
\int d \alpha^{\prime} \mathcal{M}_{\alpha \alpha^{\prime}}(\mu) \mathcal{M}_{\alpha^{\prime} \alpha^{\prime \prime}}(\mu)=\delta\left(\alpha-\alpha^{\prime \prime}\right)
$$

Namely, by expressing $\mathcal{M}_{\alpha \alpha^{\prime}}(\mu)$ from the r.h.s. of equation (4.20) substituting it in the above condition and moving shift operators to $\mathcal{M}_{\alpha^{\prime} \alpha^{\prime \prime}}(\mu)$ via integration by parts one recovers equation (4.25). This completes the derivation of our main equations which we collect below

$$
\begin{aligned}
\left(\frac{\sin \pi b(2 \alpha+\mu)}{\sin 2 \pi b \alpha} e^{\frac{b}{2} \partial_{\alpha}}+\frac{\sin \pi b(2 \alpha-\mu)}{\sin 2 \pi b \alpha} e^{-\frac{b}{2} \partial_{\alpha}}\right) \mathcal{M}_{\alpha \alpha^{\prime}}(\mu) & =2 \cos 2 \pi b \alpha^{\prime} \mathcal{M}_{\alpha \alpha^{\prime}}(\mu) \\
\left(e^{-\frac{b}{2} \partial_{\alpha^{\prime}}} \frac{\sin \pi b\left(2 \alpha^{\prime}+\mu\right)}{\sin 2 \pi b \alpha^{\prime}}+e^{\frac{b}{2} \partial_{\alpha^{\prime}}} \frac{\sin \pi b\left(2 \alpha^{\prime}-\mu\right)}{\sin 2 \pi b \alpha^{\prime}}\right) \mathcal{M}_{\alpha \alpha^{\prime}}(\mu) & =2 \cos 2 \pi b \alpha \mathcal{M}_{\alpha \alpha^{\prime}}(\mu) \\
\frac{1}{\sin 2 \pi b \alpha}\left(e^{\frac{b}{2} \partial_{\alpha}}-e^{-\frac{b}{2} \partial_{\alpha}}\right) \mathcal{M}_{\alpha \alpha^{\prime}}(\mu) & =2 e^{b \partial_{\mu}} \mathcal{M}_{\alpha \alpha^{\prime}}(\mu)
\end{aligned}
$$

In paper [7] it was shown that these equations also follow in the language of matrix models from the formalism of check operators.

Let us discuss these equations. First of them is a second-order linear difference equation with shifts in internal momentum $\alpha$. The second equation is the counterpart with shifts in the other internal momentum $\alpha^{\prime}$. It can be derived from the first and the property that $\mathcal{M}_{\alpha \alpha^{\prime}}(\mu)$ squares to unity. The third equation involves shifts in external momentum $\mu$. Hereby, we have three equations for the modular kernel which depends on three parameters.

At the first glance there are essential ambiguities in the general solution to system (4.24), (4.25), (4.26). However, as we now explain these ambiguities are artifacts of our parametrization (2.15) and can be completely removed.

Consider first equation (4.24). It is second-order linear difference homogeneous equation with $b / 2$-valued shifts in $\alpha$. Therefore, its general solution $G_{\alpha \alpha^{\prime}}(\mu)$ can be written as

$$
G_{\alpha \alpha^{\prime}}(\mu)=f_{1}\left(\underline{\alpha}, \alpha^{\prime}, \mu\right) \times S_{1}\left(\alpha, \alpha^{\prime}, \mu\right)+f_{2}\left(\underline{\alpha}, \alpha^{\prime}, \mu\right) \times S_{2}\left(\alpha, \alpha^{\prime}, \mu\right)
$$

with $f_{1}\left(\underline{\alpha}, \alpha^{\prime}, \mu\right), f_{2}\left(\underline{\alpha}, \alpha^{\prime}, \mu\right)$ arbitrary $b / 2$-periodic in $\alpha$ functions and $S_{1}(\alpha), S_{2}(\alpha)$ two independent solutions. Independence means that their ratio $S_{1} / S_{2}$ is not $b / 2$-periodic. Here and in the following we will emphasize $b / 2$-periodicity of functions w.r.t. an argument with an underline.

However, both $\alpha$ and $-\alpha$ correspond to the same conformal dimension $\Delta=\frac{Q^{2}}{4}-\alpha^{2}$. Since the modular kernel should be in fact a function of $\Delta$ we shall choose $\mathcal{M}_{\alpha \alpha^{\prime}}(\mu)$ to be an even function of $\alpha$. As we will see explicitly, this requirement picks a single solution out of two possible in (4.27). Hence, the general $\alpha$-even solution to equation (4.24) is given by

$$
\mathcal{M}_{\alpha, \alpha^{\prime}}(\mu)=f\left(\underline{\alpha}, \alpha^{\prime}, \mu\right) \times E_{\alpha \alpha^{\prime}}(\mu)
$$


with $E_{\alpha \alpha^{\prime}}(\mu)$ a particular $\alpha$-even solution which is determined up to arbitrary $\alpha$-even periodic multiplier $f\left(\underline{\alpha}, \alpha^{\prime}, \mu\right)$. By the same reasoning, from equation (4.25) we can further fix function $f\left(\underline{\alpha}, \alpha^{\prime}, \mu\right)$ up to some function $g$ which is also $b / 2$-periodic in $\alpha^{\prime}$

$$
f\left(\underline{\alpha}, \alpha^{\prime}, \mu\right) \rightarrow g\left(\underline{\alpha}, \underline{\alpha^{\prime}}, \mu\right)
$$

Finally, from equation (4.26) we constrain the dependence of $g$ on $\mu$ up to some function $h$ which is $b / 2$-periodic in all the arguments

$$
g\left(\underline{\alpha}, \underline{\alpha^{\prime}}, \mu\right) \rightarrow h\left(\underline{\alpha}, \underline{\alpha}^{\prime}, \underline{\mu}\right)
$$

By now we have seen that the seeming redundancy of solutions related to the order of equations (4.24), (4.25) is removed by demanding the solution to be an even function in $\alpha$ and $\alpha^{\prime}$. The remaining freedom in the choice of the function $h$ can also be fixed. Note that the central charge (2.15) is invariant under change $b \rightarrow b^{-1}$. As the modular kernel only depends on $c$ and not on $b$ separately, it must be invariant under $b \rightarrow b^{-1}$. This fixes undetermined function $h$ up to some other function $C$, which is both $b / 2$ and $b^{-1} / 2$-periodic in all the arguments. For generic irrational $b$ this implies that $C$ is simply a constant independent of $\alpha, \alpha^{\prime}, \mu$. Eventually, this constant can be fixed from the requirement that $\mathcal{M}_{\alpha \alpha^{\prime}}(\mu)$ squares to unity. Thus, for generic irrational values of $b$ the modular kernel is uniquely identified as the solution to equations (4.24), (4.25), (4.26) with the desired symmetry properties.

\section{Modular kernel series expansion from the difference equations}

In the present section we construct the solution to equations (4.24), (4.25), (4.26) for generic irrational $b .^{2}$ Equation (4.24) can be rewritten in the following form

$$
\begin{aligned}
\left(e^{-i \pi b \mu} e^{\frac{b}{2} \partial_{\alpha}}+e^{i \pi b \mu} e^{-\frac{b}{2} \partial_{\alpha}}-2 \cos 2 \pi b \alpha^{\prime}\right) \mathcal{M}_{\alpha \alpha^{\prime}}(\mu) & \\
& =e^{4 \pi i b \alpha}\left(e^{i \pi b \mu} e^{\frac{b}{2} \partial_{\alpha}}+e^{-i \pi b \mu} e^{-\frac{b}{2} \partial_{\alpha}}-2 \cos 2 \pi b \alpha^{\prime}\right) \mathcal{M}_{\alpha \alpha^{\prime}}(\mu)
\end{aligned}
$$

As explained in the previous section the solution to our problem with the desired symmetry properties is unique. In the sequel we construct such a solution starting with ansatz

$$
\widetilde{\mathcal{M}}_{\alpha \alpha^{\prime}}(\mu)=e^{4 \pi i \alpha \alpha^{\prime}} e^{2 \pi i \alpha \mu} \sum_{n=0}^{\infty} e^{4 \pi i n b \alpha} \mathcal{M}^{n}\left(\alpha^{\prime}, \mu\right) \times f\left(\underline{\alpha}, \alpha^{\prime}, \mu\right), \quad \mathcal{M}^{0}\left(\alpha^{\prime}, \mu\right)=1
$$

with $f\left(\underline{\alpha}, \alpha^{\prime}, \mu\right)$ yet undetermined function $b / 2$-periodic in $\alpha .^{3}$

\footnotetext{
${ }^{2}$ The difference equations for rational $b$ are also solvable. However, in these cases solutions are only determined up to a certain functional multiplier which can not be fixed from equations themselves. Hence, we omit considering such cases here.

${ }^{3}$ We remind that an underline emphasizes $b / 2$-periodicity.
} 
Plugging this ansatz into equation (5.1) results in a simple recurrence relation between coefficients $\mathcal{M}^{n}\left(\alpha^{\prime}, \mu\right)$

$$
\begin{aligned}
& \left(e^{2 \pi i b \alpha^{\prime}} e^{2 \pi i n b^{2}}+e^{-2 \pi i b \alpha^{\prime}} e^{-2 \pi i n b^{2}}-2 \cos 2 \pi b \alpha^{\prime}\right) \mathcal{M}^{n}\left(\alpha^{\prime}, \mu\right) \\
& \quad=\left(e^{2 \pi i b \alpha^{\prime}} e^{2 \pi i b \mu} e^{2 \pi i(n-1) b^{2}}+e^{-2 \pi i b \alpha^{\prime}} e^{-2 \pi i b \mu} e^{-2 \pi i(n-1) b^{2}}-2 \cos 2 \pi b \alpha^{\prime}\right) \mathcal{M}^{n-1}\left(\alpha^{\prime}, \mu\right)
\end{aligned}
$$

Solving this relation and making use of the elementary trigonometric identities gives

$$
\mathcal{M}^{n}\left(\alpha^{\prime}, \mu\right)=\prod_{k=1}^{n} \frac{\sin \pi b\left(2 \alpha^{\prime}+\mu+(k-1) b\right) \sin \pi b(\mu+(k-1) b)}{\sin \pi b\left(2 \alpha^{\prime}+k b\right) \sin \pi k b^{2}}
$$

The next step is to partially fix function $f\left(\underline{\alpha}, \alpha^{\prime}, \mu\right)$ by imposing that our ansatz (5.2) also satisfies equation (4.25). The simplest way to do it is by noting a symmetry between equations (4.24) and (4.25). Namely, as can be seen through direct manipulations, if some function $m_{\alpha \alpha^{\prime}}(\mu)$ solves equation (4.24) then function $m_{\alpha^{\prime} \alpha}(b-\mu) \times \sin 2 \pi b \alpha^{\prime} \times r\left(\alpha, \underline{\alpha^{\prime}}, \mu\right)$ with any function $r$ which is $b / 2$-periodic in $\alpha^{\prime}$ solves equation (4.25). Consequently, if some function $m_{\alpha \alpha^{\prime}}(\mu)$ solves equation (4.24) and satisfies

$$
\frac{m_{\alpha^{\prime} \alpha}(b-\mu) \times \sin 2 \pi b \alpha^{\prime}}{m_{\alpha \alpha^{\prime}}(\mu)}=r\left(\alpha, \underline{\alpha^{\prime}}, \mu\right)
$$

then such a function solves both equations (4.24), (4.25). We can choose function $f\left(\underline{\alpha}, \alpha^{\prime}, \mu\right)$ so that our ansatz (5.2) fulfills condition (5.5). A key observation is that

$$
\sum_{n=0}^{\infty} e^{4 \pi i n b \alpha} \mathcal{M}^{n}\left(\alpha^{\prime}, \mu\right)=\left(1-e^{4 \pi i b \alpha^{\prime}}\right) \frac{\widetilde{S}\left(2 \alpha^{\prime} \mid b\right)}{\widetilde{S}\left(2 \alpha^{\prime}+\mu \mid b\right)} \mathcal{N}_{\alpha \alpha^{\prime}}(\mu)
$$

where function $\widetilde{S}$ is defined in appendix A, (A.10) and function $\mathcal{N}_{\alpha \alpha^{\prime}}(\mu)$ is given by

$$
\mathcal{N}_{\alpha \alpha^{\prime}}(\mu)=\sum_{n, m=0}^{\infty} e^{4 \pi i n b \alpha} e^{4 \pi i m b \alpha^{\prime}} e^{2 \pi i b^{2} n m} \prod_{k=1}^{n} \frac{e^{2 \pi i k b^{2}}-e^{2 \pi i b(b-\mu)}}{e^{2 \pi i k b^{2}}-1} \prod_{l=1}^{m} \frac{e^{2 \pi i l b^{2}}-e^{2 \pi i b \mu}}{e^{2 \pi i l b^{2}}-1}
$$

Relation (5.6) is proven in appendix B. Function $\mathcal{N}_{\alpha \alpha^{\prime}}(\mu)$ is manifestly symmetric under the transformation of interest $\mathcal{N}_{\alpha \alpha^{\prime}}(\mu)=\mathcal{N}_{\alpha^{\prime} \alpha}(b-\mu)$. Therefore, we obtain

$$
\begin{aligned}
& \frac{\widetilde{M}_{\alpha^{\prime} \alpha}(b-\mu) \times \sin 2 \pi b \alpha^{\prime}}{\widetilde{M}_{\alpha \alpha^{\prime}}(\mu)}=\left(\frac{1}{f\left(\underline{\alpha}, \alpha^{\prime}, \mu\right)}\right.\left.\sin 2 \pi b \alpha^{\prime} \frac{e^{2 \pi i \alpha^{\prime}(b-\mu)}}{1-e^{4 \pi i b \alpha^{\prime}}} \frac{\widetilde{S}\left(2 \alpha^{\prime}+\mu \mid b\right)}{\widetilde{S}\left(2 \alpha^{\prime} \mid b\right)}\right) \\
& \times\left(f\left(\underline{\alpha}^{\prime}, \alpha, b-\mu\right) \frac{1-e^{4 \pi i b \alpha}}{e^{2 \pi i \alpha \mu}} \frac{\widetilde{S}(2 \alpha \mid b)}{\widetilde{S}(2 \alpha+\mu \mid b)}\right)
\end{aligned}
$$

The last factor in braces is already $b / 2$-periodic in $\alpha^{\prime}$. We can render the first factor periodic as well by choosing

$$
f\left(\underline{\alpha}, \alpha^{\prime}, \mu\right)=e^{-2 \pi i \alpha^{\prime} \mu} \frac{\widetilde{S}\left(2 \alpha^{\prime}+\mu \mid b\right)}{\widetilde{S}\left(2 \alpha^{\prime} \mid b\right)} \times g\left(\underline{\alpha}, \underline{\alpha^{\prime}}, \mu\right)
$$

With some function $g\left(\underline{\alpha}, \underline{\alpha}^{\prime}, \mu\right)$ which is $b / 2$-periodic in both $\alpha$ and $\alpha^{\prime}$. 
It remains to fix $\mu$ dependence of function $g\left(\underline{\alpha}, \underline{\alpha^{\prime}}, \mu\right)$ by imposing that our ansatz (5.2) with function $f\left(\underline{\alpha}, \alpha^{\prime}, \mu\right)$ given by formula (5.9) satisfies equation (4.26). It appears that only a simple phase factor has to be included in $g\left(\underline{\alpha}, \underline{\alpha}^{\prime}, \mu\right)$ in order for our ansatz to also satisfy equation (4.26)

$$
g\left(\underline{\alpha}, \underline{\alpha^{\prime}}, \mu\right)=e^{\frac{i \pi}{2} \mu(Q-\mu)} h\left(\underline{\alpha}, \underline{\alpha^{\prime}}, \underline{\mu}\right)
$$

Here $h$ is any function $b / 2$-periodic in all the arguments. Proving that with such a choice of $g$ our ansatz satisfies (4.26) is straightforward but a little bulky and we relegate it to appendix $\mathrm{C}$.

Now, as we have a function that satisfies all of the equations (4.24), (4.25), (4.26), the last two steps are due. First, we should impose the self-duality condition, i.e. the symmetry of $\widetilde{\mathcal{M}}_{\alpha \alpha^{\prime}}(\mu)$ under change $b \rightarrow b^{-1}$. This is implemented trivially by choosing

$$
\begin{aligned}
& h\left(\underline{\alpha}, \underline{\alpha^{\prime}}, \underline{\mu}\right)=\frac{\widetilde{S}\left(2 \alpha^{\prime}+\mu \mid b^{-1}\right)}{\widetilde{S}\left(2 \alpha^{\prime} \mid b^{-1}\right)} \\
& \times \sum_{n=0}^{\infty} e^{4 \pi i n b^{-1} \alpha} \prod_{k=1}^{n} \frac{\sin \pi b^{-1}\left(2 \alpha^{\prime}+\mu+(k-1) b^{-1}\right) \sin \pi b^{-1}\left(\mu+(k-1) b^{-1}\right)}{\sin \pi b^{-1}\left(2 \alpha^{\prime}+k b^{-1}\right) \sin \pi k b^{-2}} \times C
\end{aligned}
$$

where $C$ is both $b / 2$ and $b^{-1} / 2$-periodic in all the arguments and hence is simply a constant for generic irrational values of $b$. Second, we should construct an even in $\alpha, \alpha^{\prime}$ solution out of our particular solution (5.2). Recall that our solution is a series expansion in powers of $e^{4 \pi i b \alpha}, e^{4 \pi i b^{-1} \alpha}$. If such an expansion is convergent for some $\alpha$ (parameters $e^{4 \pi i b \alpha}, e^{4 \pi i b^{-1} \alpha}$ are small) then it typically is divergent for the opposite value $\widetilde{\alpha}=-\alpha$ (parameters $e^{4 \pi i b \widetilde{\alpha}}, e^{4 \pi i b^{-1} \widetilde{\alpha}}$ are large). Hence we simply define our function for these values as an even extension from the region of convergence. Note that since each of equations $(4.24),(4.25),(4.26)$ are symmetric w.r.t. $\alpha \rightarrow-\alpha$ the extended function is a solution everywhere where it is defined. For symmetrization in $\alpha^{\prime}$ we simply add the same function that we already have with $\alpha^{\prime}$ replaced by $-\alpha^{\prime}$. Thus, the particular solution respecting all the symmetries in play can be written as

$$
\mathcal{M}_{\alpha \alpha^{\prime}}(\mu)=\widetilde{\mathcal{M}}_{\alpha \alpha^{\prime}}(\mu)+\widetilde{\mathcal{M}}_{\alpha,-\alpha^{\prime}}(\mu)
$$

And it is only left to fix the overall normalization $C$ requiring that $\mathcal{M}$ squares to unity. This condition is most easily verified for $\mu=0$ when $\widetilde{\mathcal{M}}_{\alpha \alpha^{\prime}}(0)=C e^{4 \pi i \alpha \alpha^{\prime}}$ and $\mathcal{M}_{\alpha \alpha^{\prime}}(0)=$ $2 C \cos 4 \pi \alpha \alpha^{\prime}$. A simple calculation then shows that

$$
C=2^{-1 / 2}
$$

This leads us to the final expression for a part of the modular kernel $\widetilde{\mathcal{M}}_{\alpha \alpha^{\prime}}(\mu)$

$$
\begin{aligned}
& \widetilde{\mathcal{M}}_{\alpha \alpha^{\prime}}(\mu)=2^{-1 / 2} e^{4 \pi i \alpha \alpha^{\prime}} e^{2 \pi i \mu \alpha} e^{i \pi \mu(Q-\mu) / 2} \frac{S_{b}\left(2 \alpha^{\prime}+\mu\right)}{S_{b}\left(2 \alpha^{\prime}\right)} \\
& \quad \times\left(\sum_{n=0}^{\infty} e^{4 \pi i n b \alpha} \prod_{k=1}^{n} \frac{\sin \pi b\left(2 \alpha^{\prime}+\mu+(k-1) b\right) \sin \pi b(\mu+(k-1) b)}{\sin \pi b\left(2 \alpha^{\prime}+k b\right) \sin \pi k b^{2}}\right) \times\left(b \rightarrow b^{-1}\right)
\end{aligned}
$$


Here only the sum in the second line (in braces) is multiplied by its counterpart with $b \rightarrow b^{-1}$. The full modular kernel is constructed by symmetrization in $\alpha^{\prime}$ according to equation (5.12).

Alternatively, we can use representation (5.6) and the following straightforward relation (see definitions in appendix A)

$$
\frac{S_{b}\left(2 \alpha^{\prime}+\mu\right)}{S_{b}\left(2 \alpha^{\prime}\right)} \frac{\widetilde{S}\left(2 \alpha^{\prime} \mid b\right) \widetilde{S}\left(2 \alpha^{\prime} \mid b^{-1}\right)}{\widetilde{S}\left(2 \alpha^{\prime}+\mu \mid b\right) \widetilde{S}\left(2 \alpha^{\prime}+\mu \mid b^{-1}\right)}=\frac{S_{0}\left(2 \alpha^{\prime}+\mu \mid Q\right)}{S_{0}\left(2 \alpha^{\prime} \mid Q\right)}=e^{i \pi \mu(Q-\mu) / 2} e^{-2 \pi i \alpha^{\prime} \mu}
$$

to rewrite $\widetilde{\mathcal{M}}_{\alpha \alpha^{\prime}}(\mu)$ in a more symmetric double-expanded form

$$
\begin{aligned}
& \widetilde{\mathcal{M}}_{\alpha \alpha^{\prime}}(\mu)=2^{3 / 2} e^{4 \pi i \alpha \alpha^{\prime}} e^{2 \pi i\left(\mu \alpha+(Q-\mu) \alpha^{\prime}\right)} e^{i \pi \mu(Q-\mu)} \sin 2 \pi b \alpha^{\prime} \sin 2 \pi b^{-1} \alpha^{\prime} \\
& \quad \times \sum_{n, m=0}^{\infty} e^{4 \pi i n b \alpha} e^{4 \pi i m b \alpha^{\prime}} e^{2 \pi i n m b^{2}} \prod_{k=1}^{n} \frac{e^{2 \pi i k b^{2}}-e^{2 \pi i b(b-\mu)}}{e^{2 \pi i k b^{2}}-1} \prod_{l=1}^{m} \frac{e^{2 \pi i l b^{2}}-e^{2 \pi i b \mu}}{e^{2 \pi i l b^{2}}-1} \times\left(b \rightarrow b^{-1}\right)
\end{aligned}
$$

We conclude this section with several comments on formulas (5.14), (5.16).

1) The series expansions of the modular kernel here are presented in a special basis of conformal blocks, with normalization different from standard (2.12), (2.14). The exact relation is spelled in equation (4.17).

2) By construction this modular kernel is symmetric w.r.t. the following reflections

$$
\alpha \rightarrow-\alpha, \quad \alpha^{\prime} \rightarrow-\alpha^{\prime}
$$

In contrast, the symmetry $\mu \rightarrow Q-\mu$ is broken in the normalization we use (4.17).

According to our knowledge, there is no a-priori symmetry properties that the modular kernel must have under the exchange of internal dimensions $\alpha \leftrightarrow \alpha^{\prime}$. However, it is easy to see explicitly from expression (5.16) that in the normalization used the following relation holds

$$
\frac{\mathcal{M}_{\alpha^{\prime} \alpha}(\mu)}{\sin 2 \pi b \alpha \sin 2 \pi b^{-1} \alpha}=\frac{\mathcal{M}_{\alpha \alpha^{\prime}}(Q-\mu)}{\sin 2 \pi b \alpha^{\prime} \sin 2 \pi b^{-1} \alpha^{\prime}}
$$

3) The series representations are valid in any domain of the parameter space where they are convergent. For example, the default setup in the Liouville theory is $c \geq 1$ restricting $Q \in \mathbb{R}$ while $\Delta \geq(c-1) / 24 \in \mathbb{R}$ imposing $\alpha \in i \mathbb{R}, \mu \in \frac{Q}{2}+i \mathbb{R}$. Then, the series expansions are convergent for $b \in \mathbb{R}, \alpha \in i \mathbb{R}_{+}$. In fact, the domain of validity of the series representation seems to be the same as for the integral formula. For instance, for the minimal models corresponding to $c<1$ we have to pick imaginary $b$ and then exponents $e^{4 \pi i n b \alpha}$ refuse to be small rendering the series not convergent.

4) Note that one can not directly use the double-expansion (5.16) in equation (5.12) since if $\widetilde{\mathcal{M}}_{\alpha \alpha^{\prime}}$ is convergent then $\widetilde{\mathcal{M}}_{\alpha,-\alpha^{\prime}}$ will diverge for the same $\alpha^{\prime}$. Instead, an analytic continuation in one of the terms should be implied. 
5) The necessity to add a second term with $\alpha^{\prime} \rightarrow-\alpha^{\prime}$ in formula (5.12) is somewhat formal. Since the conformal block can only depend on $\alpha$ through a conformal dimension $\Delta=Q^{2} / 4-\alpha^{2}$ it must be an even function of momentum $\alpha$. Therefore, for the transformations of the conformal blocks $\alpha^{\prime}$-odd part of the modular kernel is not essential.

\section{Integral representation of the toric modular kernel}

In paper [2] formula for the modular kernel of the generic toric conformal block was presented in the following integral form ${ }^{4}$

$$
\mathcal{M}_{\alpha \alpha^{\prime}}(\mu)=\frac{2^{3 / 2}}{i} \frac{\sin 2 \pi b \alpha^{\prime} \sin 2 \pi b^{-1} \alpha^{\prime}}{S_{b}(\mu)} \int_{\mathcal{C}} d \xi \frac{S_{b}\left(\alpha^{\prime}+\frac{\mu}{2}+\xi\right) S_{b}\left(\alpha^{\prime}+\frac{\mu}{2}-\xi\right)}{S_{b}\left(\alpha^{\prime}+Q-\frac{\mu}{2}+\xi\right) S_{b}\left(\alpha^{\prime}+Q-\frac{\mu}{2}-\xi\right)} e^{-4 \pi i \alpha \xi}
$$

Here $S_{b}(z)$ is a special function we call the double sine function. It is described in appendix A. This formula is written in the same normalization as series expansions (5.14), (5.16).

For generic $c>1$ integrand in (6.1) contains four infinite families of poles lying on halflines and integration contour $\mathcal{C}$ is chosen to maneuver between them in a specific way. For certain values of parameters some of these poles can overlap and merge and formula (6.1) needs a completion. In some cases this issue is not resolvable. For example, for $c \leq 1$ (and thus for all minimal models) the formula is not valid.

We now derive the series expansion (5.14) directly from the integral representation (6.1). As turns out, the series expansion corresponds to the sum over the residues of the integral. Function $S_{b}(z)$ is meromorphic with simple poles and zeros located at

$$
\begin{array}{ll}
\text { zeros : } z=n b+m b^{-1}, & n, m \geq 1 \\
\text { poles : } z=-n b-m b^{-1}, & n, m \geq 0
\end{array}
$$

Therefore, the integrand of (6.1) has simple poles at the points

$$
\begin{aligned}
& \xi_{I I}^{(n, m)}=\alpha^{\prime}+Q-\mu / 2-n b-m b^{-1}, \quad n, m \geq 1 \\
& \xi_{I}^{(n, m)}=\alpha^{\prime}+\mu / 2+n b+m b^{-1}, \quad n, m \geq 0 \\
& \xi_{I I I}^{(n, m)}=-\alpha^{\prime}-\mu / 2-n b-m b^{-1}, \quad n, m \geq 0 \\
& \xi_{I V}^{(n, m)}=-\alpha^{\prime}-Q+\mu / 2+n b+m b^{-1}, \quad n, m \geq 1
\end{aligned}
$$

\footnotetext{
${ }^{4}$ We warn the reader of notational difference with the original paper. The exact relation is $\alpha=i p_{a}, \alpha^{\prime}=$ $i p_{b}, \mu=Q / 2+i p_{e}, c_{b}=i Q / 2, s_{b}(z)=S_{b}(Q / 2+i z)$.
} 
We can depict them in the complex $\xi$-plane



Possible integration contour $\mathcal{C}$ in (6.1) is shown by the blue line. Assume now that $\alpha \in i \mathbb{R}_{+}$ and $b>0$. Then exponent $e^{-4 \pi i \alpha \xi}$ in (6.1) decays for $\Re \xi<0$ and the integral can be represented as the sum over the residues collected at $z \in \xi_{I I}$ and $z \in \xi_{I I I}$.

Note that this figure is a little schematic. In each of the four families poles are not located equidistantly; for complex values of $b$ one would have wedges instead of half-lines as the domains containing poles; in some circumstances the four groups are not sharply separated and the contour of integration can not be chosen to run along the imaginary axes (we have shifted the contour at the figure away from imaginary axis for the clarity of the picture). However, no matter what the deformation is necessary to correct the figure in any particular case, the result remains simple: we can compute integral (6.1) accounting for residues $\xi_{I I}$ and $\xi_{I I I}$.

Denote the integrand of (6.1) by $\mathcal{I}$, then

$$
\begin{aligned}
& 2 \pi i \operatorname{Res}_{\xi_{I I I}^{(n, m)}} \mathcal{I} \\
& \quad=2 \pi i \frac{S_{b}\left(2 \alpha^{\prime}+\mu+n b+m b^{-1}\right) S_{b}\left(\mu+n b+m b^{-1}\right)}{S_{b}\left(2 \alpha^{\prime}+Q+n b+m b^{-1}\right) \operatorname{Res}^{-1} S_{b}\left(-n b-m b^{-1}\right)} e^{4 \pi i \alpha \alpha^{\prime}} e^{2 \pi i \alpha \mu} e^{4 \pi i \alpha\left(n b+m b^{-1}\right)}
\end{aligned}
$$

From definition (6.3) one sees that the poles corresponding to $\xi_{I I}$ describe the same set as $\xi_{I I I}$ but with $\alpha^{\prime}$ replaced by $-\alpha^{\prime}$ (recall that $Q=b+b^{-1}$ ). Also, the integrand is $\alpha^{\prime}$-even function as can be seen from property (A.4). As a consequence the residues of the integrand at $\xi_{I I}$ are exactly the same as at $\xi_{I I I}$ with $\alpha^{\prime}$ replaced by $-\alpha^{\prime}$. Thus we can only consider the sum over $\xi_{I I I}$ residues and then symmetrize the result to get an even function of $\alpha^{\prime}$. 
Using property (A.5) and explicit form of residues (A.7) we can rewrite the obtained expression in the following way

$$
\begin{aligned}
2 \pi i \operatorname{Res}_{\xi_{I I I}^{(n, m)}} \mathcal{I}= & i e^{4 \pi i \alpha \alpha^{\prime}} e^{2 \pi i \alpha \mu} e^{4 \pi i \alpha\left(n b+m b^{-1}\right)} S_{b}(\mu) \frac{S_{b}\left(2 \alpha^{\prime}+\mu\right)}{S_{b}\left(2 \alpha^{\prime}+Q\right)} \\
& \times \prod_{k=1}^{n} \frac{\sin \pi b\left(2 \alpha^{\prime}+\mu+(k-1) b\right) \sin \pi b(\mu+(k-1) b)}{\sin \pi b\left(2 \alpha^{\prime}+k b\right) \sin \pi b^{2} k} \\
& \times \prod_{l=1}^{m} \frac{\sin \pi b^{-1}\left(2 \alpha^{\prime}+\mu+(l-1) b^{-1}\right) \sin \pi b^{-1}\left(\mu+(l-1) b^{-1}\right)}{\sin \pi b^{-1}\left(2 \alpha^{\prime}+l b^{-1}\right) \sin \pi b^{-2} l}
\end{aligned}
$$

Finally, noting that

$$
\frac{2^{3 / 2} \sin 2 \pi b \alpha^{\prime} \sin 2 \pi b^{-1} \alpha^{\prime}}{i} \frac{i}{S_{b}\left(2 \alpha^{\prime}+Q\right)}=\frac{2^{-1 / 2}}{S_{b}\left(2 \alpha^{\prime}\right)}
$$

we see, that summing up all the contributions from $\xi_{I I I}$ and $\xi_{I I}$ groups of poles exactly reproduces formula (5.14). We thus conclude that expressions (5.14), (5.16) indeed serve as series representations to the original integral expression (6.1).

Note that for $\alpha \in-i \mathbb{R}_{+}$we can enclose the integration contour in (6.1) in the right half-plane collecting $\xi_{I}$ and $\xi_{I V}$ residues. As is seen from (6.3) and the symmetry of the integrand the result will be exactly the same up to replacement $\alpha \rightarrow-\alpha$. This simply means that $\mathcal{M}_{\alpha \alpha^{\prime}}(\mu)$ is even w.r.t. $\alpha$, as it should be.

\section{Conclusion}

The main result of the paper are formulas (5.14), (5.16) for the toric modular kernel of non-degenerate one-point toric Virasoro conformal blocks. These formulas are to be considered as series expansions of the integral expression originally presented in paper [2]. It is worth mentioning that due to paper [8] there exists an alternative representation for the toric modular kernel involving the fusion matrix of the spheric conformal blocks first presented in $[9,10]$. Also, in work [11] it was demonstrated that the integral expressions also satisfy the full set of the consistency conditions, not only those containing the degenerate operators.

Besides giving yet another representation for the modular kernel which may prove useful, our formulas reveal a curious structure. Namely, they take the form of a series expansion in parameters

$$
e^{4 \pi i b^{ \pm 1} \alpha}, e^{4 \pi i b^{ \pm 1} \alpha^{\prime}}
$$

on top of the simple Fourier-kernel

$$
e^{4 \pi i \alpha \alpha^{\prime}}
$$

Such a structure does not seem natural from the conformal blocks point of view and parameters (7.1) do not appear in usual considerations. In fact, it was shown recently 
in [12-15] that perturbatively modular transformations of conformal blocks are nothing but the Fourier transformation. Therefore, expressions (5.14), (5.16) may be interpreted as nonperturbative expansions about this asymptotic Fourier form. The method to obtain these non-perturbative series was indirect and explicitly used only properties of the degenerate conformal blocks. To test these formulas against the the non-degenerate conformal blocks and explain the origin of these non-perturbative corrections seems to be important and open issue.

\section{Acknowledgments}

The author is grateful to all the people he has engaged in discussions on the subject and especially to Alexei Morozov and Andrei Mironov for their great assistance. I am also indebted to Sylvain Ribault for his in-depth reading of the manuscript and suggesting a lot of essential improvements. The work is partly supported by grant NSh-1500.2014.2, by RFBR 13-02-00457-a, by RFBR 14-02-31372 mol a, by 15 \&\#8722; 51 \&\#8722; 52031NSC-a, by 15-51-50041-YaF, and by D. Zimin's "Dynasty" foundation stipend.

\section{A Special functions}

Double gamma function $\Gamma_{b}(z)$ can be defined by means of the integral representation

$$
\log \Gamma_{b}(z)=\int_{0}^{\infty} \frac{d t}{t}\left(\frac{e^{-z t}-e^{-Q t / 2}}{\left(1-e^{-b t}\right)\left(1-e^{-b^{-1} t}\right)}-\frac{(Q-2 z)^{2}}{8 e^{t}}-\frac{Q-2 z}{2 t}\right), \quad Q=b+b^{-1}
$$

For our purposes the main property of this function is difference relation

$$
\Gamma_{b}(z+b)=\frac{\sqrt{2 \pi} b^{b z-1 / 2}}{\Gamma(b z)} \Gamma_{b}(z)
$$

The double sine function $S_{b}(z)$ can be defined via

$$
S_{b}(z)=\frac{\Gamma_{b}(z)}{\Gamma_{b}(Q-z)}
$$

From the definition one derives

$$
\begin{aligned}
S_{b}(Q-z) & =\frac{1}{S_{b}(z)} \\
S_{b}(z+b) & =2 \sin \pi b z S_{b}(z)
\end{aligned}
$$

Note that

$$
\Gamma_{b}(z)=\Gamma_{b^{-1}}(z), \quad S_{b}(z)=S_{b^{-1}}(z)
$$

This property is often called self-duality. 
Double sine function has poles at points $z=-n b-m b^{-1}(n, m \geq 0)$ and zeros at the points $z=n b+m b^{-1}(n, m \geq 1)$. The corresponding residues are

$$
\begin{aligned}
\operatorname{Res} S_{b}\left(-n b-m b^{-1}\right) & =\frac{1}{2 \pi} \frac{(-1)^{n m+n+m}}{\prod_{k=1}^{n} 2 \sin \pi k b^{2} \prod_{l=1}^{m} 2 \sin \pi l b^{-2}} \\
\operatorname{Res} S_{b}^{-1}\left(n b+m b^{-1}\right) & =\frac{1}{2 \pi} \frac{(-1)^{n m}}{\prod_{k=1}^{n-1} 2 \sin \pi k b^{2} \prod_{l=1}^{m-1} 2 \sin \pi l b^{-2}}
\end{aligned}
$$

The following series representation of $S_{b}$ is of a great use for our purposes

$$
\log S_{b}(z)=-\frac{i \pi}{2}\left(z^{2}-Q z+\frac{Q^{2}+1}{6}\right)-\sum_{n=1}^{\infty} \frac{1}{n} \frac{e^{2 \pi i n b z}}{e^{2 \pi i n b^{2}}-1}-\sum_{n=1}^{\infty} \frac{1}{n} \frac{e^{2 \pi i n b^{-1} z}}{e^{2 \pi i n b^{-2}}-1}
$$

It is also convenient to introduce notation

$$
\begin{aligned}
\log S_{0}(z \mid Q) & =-\frac{i \pi}{2}\left(z^{2}-Q z+\frac{Q^{2}+1}{6}\right) \\
\log \widetilde{S}(z \mid b) & =-\sum_{n=1}^{\infty} \frac{1}{n} \frac{e^{2 \pi i n b z}}{e^{2 \pi i n b^{2}}-1}
\end{aligned}
$$

so that the double sine function is split into three factors

$$
S_{b}(z)=S_{0}(z \mid Q) \widetilde{S}(z \mid b) \widetilde{S}\left(z \mid b^{-1}\right)
$$

One has

$$
\frac{\widetilde{S}(z+b \mid b)}{\widetilde{S}(z \mid b)}=1-e^{2 \pi i b z}
$$

This completes the list of the properties which we use in the article.

\section{B Identity relating double and single expansions of the modular kernel}

In this appendix we prove identity (5.6) which is equivalent to confirming the following formula

$$
L(\boldsymbol{p}, \mu)=R(\boldsymbol{p}, \mu)
$$

where

$$
\begin{gathered}
L(\boldsymbol{p}, \mu)=\prod_{k=1}^{n} \frac{\sin \pi b\left(2 \alpha^{\prime}+\mu+(k-1) b\right) \sin \pi b(\mu+(k-1) b)}{\sin \pi b\left(2 \alpha^{\prime}+k b\right) \sin \pi k b^{2}} \\
R(\boldsymbol{p}, \mu)=\left(1-e^{4 \pi i b \alpha^{\prime}}\right) \frac{\widetilde{S}\left(2 \alpha^{\prime} \mid b\right)}{\widetilde{S}\left(2 \alpha^{\prime}+\mu \mid b\right)} \prod_{k=1}^{n} \frac{e^{2 \pi i k b^{2}}-e^{2 \pi i b(b-\mu)}}{e^{2 \pi i k b^{2}}-1} \\
\times \sum_{m=0}^{\infty} e^{4 \pi i m b \alpha^{\prime}} e^{2 \pi i b^{2} n m} \prod_{l=1}^{m} \frac{e^{2 \pi i l b^{2}}-e^{2 \pi i b \mu}}{e^{2 \pi i l b^{2}}-1}
\end{gathered}
$$

and $\boldsymbol{p}$ denotes collectively all the other parameters except $\mu, \boldsymbol{p}=\left(\alpha^{\prime}, b, n\right)$. 
We first note that when $\mu=b$ one has $L(\boldsymbol{p}, b)=1$ and due to relation (A.12) also $R(\boldsymbol{p}, b)=1$, i.e.

$$
L(\boldsymbol{p}, b)=R(\boldsymbol{p}, b)
$$

Next, one easily sees that both functions satisfy the same difference equation of the first order with $b^{-1}$-valued shift in $\mu$

$$
e^{b^{-1} \partial_{\mu}} L(\boldsymbol{p}, \mu)=L(\boldsymbol{p}, \mu), \quad e^{b^{-1} \partial_{\mu}} R(\boldsymbol{p}, \mu)=R(\boldsymbol{p}, \mu)
$$

This implies that

$$
L(\boldsymbol{p}, \mu)=R(\boldsymbol{p}, \mu) \times f(\boldsymbol{p}, \mu)
$$

where $f(\boldsymbol{p}, \mu)$ is some function $b$-periodic in $\mu$. As we demonstrate in the rest of this appendix, functions $L(\boldsymbol{p}, \mu)$ and $R(\boldsymbol{p}, \mu)$ also satisfy the same first-order difference equation with $b$-valued shift in $\mu$. This means that function $f(\boldsymbol{p}, \mu)$ is both $b$ and $b^{-1}$ periodic in $\mu$ and hence does not depend on $\mu$ at all (for generic irrational $b$ )

$$
f(\boldsymbol{p}, \mu)=g(\boldsymbol{p})
$$

On the other hand, since $L(\boldsymbol{p}, b)=R(\boldsymbol{p}, b)$ we conclude that $g(\boldsymbol{p})=1$ and therefore equality (B.1) holds. It now remains to demonstrate that both functions do satisfy the same difference equation with $b$-valued shift in $\mu$.

One easily sees that

$$
e^{b \partial_{\mu}} L(\boldsymbol{p}, \mu)=\Lambda(\boldsymbol{p}, \mu) L(\boldsymbol{p}, \mu)
$$

where

$$
\Lambda(\boldsymbol{p}, \mu)=\frac{\sin \pi b\left(2 \alpha^{\prime}+\mu+n b\right) \sin \pi b(\mu+n b)}{\sin \pi b\left(2 \alpha^{\prime}+\mu\right) \sin \pi b \mu}
$$

Next, using relation (A.12) and equalities

$$
\begin{aligned}
e^{b \partial_{\mu}} \prod_{k=1}^{n} \frac{e^{2 \pi i k b^{2}}-e^{2 \pi i b(b-\mu)}}{e^{2 \pi i k b^{2}}-1} & =\frac{1-e^{-2 \pi i n b^{2}} e^{-2 \pi i b \mu}}{1-e^{-2 \pi i b \mu}} \prod_{k=1}^{n} \frac{e^{2 \pi i k b^{2}}-e^{2 \pi i b(b-\mu)}}{e^{2 \pi i k b^{2}}-1} \\
e^{b \partial_{\mu}} \prod_{l=1}^{m} \frac{e^{2 \pi i l b^{2}}-e^{2 \pi i b \mu}}{e^{2 \pi i l b^{2}}-1} & =\frac{1-e^{2 \pi i b \mu}}{1-e^{-2 \pi i m b^{2}} e^{2 \pi i b \mu}} \prod_{l=1}^{m} \frac{e^{2 \pi i l b^{2}}-e^{2 \pi i b \mu}}{e^{2 \pi i l b^{2}}-1}
\end{aligned}
$$

one shows that

$$
\begin{aligned}
e^{b \partial_{\mu}} R(\boldsymbol{p}, \mu)=\{ & \left.\frac{1-e^{-2 \pi i n b^{2}} e^{-2 \pi i b \mu}}{1-e^{2 \pi i b\left(2 \alpha^{\prime}+\mu\right)}} \frac{1}{1-e^{-2 \pi i b \mu}}\right\} \\
& \times\left(1-e^{4 \pi i b \alpha^{\prime}}\right) \frac{\widetilde{S}\left(2 \alpha^{\prime} \mid b\right)}{\widetilde{S}\left(2 \alpha^{\prime}+\mu \mid b\right)} \prod_{k=1}^{n} \frac{e^{2 \pi i k b^{2}}-e^{2 \pi i b(b-\mu)}}{e^{2 \pi i k b^{2}}-1} \\
& \times \sum_{m=0}^{\infty} e^{4 \pi i m b \alpha^{\prime}}\left[\frac{e^{2 \pi i b \mu}-1}{1-e^{2 \pi i b \mu} e^{-2 \pi i m b^{2}}}\right] e^{2 \pi i n m b^{2}} \prod_{l=1}^{m} \frac{e^{2 \pi i l b^{2}}-e^{2 \pi i b \mu}}{e^{2 \pi i l b^{2}}-1}
\end{aligned}
$$


Thus, the effect of the $b$-valued shift in $\mu$ on $R(\boldsymbol{p}, \mu)$ is to multiply the whole expression by the factor in the first line (in curly brackets) and to multiply each coefficient in the series by the corresponding term (in square brackets). We now show that the multiplication by $\Lambda(\boldsymbol{p}, \mu)(\mathrm{B} .9)$ leads to the same result. Let us rewrite $\Lambda(\boldsymbol{p}, \mu)$ as

$$
\Lambda(\boldsymbol{p}, \mu)=\left\{\frac{1-e^{-2 \pi i n b^{2}} e^{-2 \pi i b \mu}}{1-e^{2 \pi i b\left(2 \alpha^{\prime}+\mu\right)}} \frac{1}{1-e^{-2 \pi i b \mu}}\right\} \times\left(1-e^{4 \pi i b \alpha^{\prime}} e^{2 \pi i b \mu} e^{2 \pi i n b^{2}}\right)
$$

Here the factor in curly brackets is the same as in equation (B.12). Let us denote

$$
R^{m}=e^{2 \pi i b^{2} n m} \prod_{l=1}^{m} \frac{e^{2 \pi i l b^{2}}-e^{2 \pi i b \mu}}{e^{2 \pi i l b^{2}}-1}
$$

Then

$$
\begin{aligned}
& \left(1-e^{4 \pi i b \alpha^{\prime}} e^{2 \pi i b \mu} e^{2 \pi i n b^{2}}\right) \sum_{m=0}^{\infty} e^{4 \pi i n b \alpha^{\prime}} R^{m} \\
& =\sum_{m=0}^{\infty} e^{4 \pi i n b \alpha^{\prime}} R^{m}\left[1-e^{2 \pi i b \mu} e^{2 \pi i n b^{2}} \frac{R^{m-1}}{R^{m}}\right] \\
& =\sum_{m=0}^{\infty} e^{4 \pi i n b \alpha^{\prime}} R^{m}\left[1-e^{2 \pi i b \mu} e^{2 \pi i n b^{2}} e^{-2 \pi i n b^{2}} \frac{e^{2 \pi i m b^{2}}-1}{e^{2 \pi i m b^{2}}-e^{2 \pi i b \mu}}\right] \\
& =\sum_{m=0}^{\infty} e^{4 \pi i n b \alpha^{\prime}} R^{m}\left[\frac{1-e^{2 \pi i b \mu}}{1-e^{2 \pi i b \mu} e^{-2 \pi i n b^{2}}}\right]
\end{aligned}
$$

The factor in square brackets here is the same as in formula (B.12). Hence, the multiplication by $\Lambda(\boldsymbol{p}, \mu)(\mathrm{B} .9)$ is indeed the same as shifting $\mu$ by $b$ in $R(\boldsymbol{p}, \mu)$. Therefore function $R(\boldsymbol{p}, \mu)$ satisfies

$$
e^{b \partial_{\mu}} R(\boldsymbol{p}, \mu)=\Lambda(\boldsymbol{p}, \mu) R(\boldsymbol{p}, \mu)
$$

which according to our previous reasoning proves equality (B.1).

\section{Verifying difference equation with shift in $\mu$}

In this appendix we show that function

$$
\widetilde{\mathcal{M}}_{\alpha \alpha^{\prime}}(\mu)=e^{4 \pi i \alpha \alpha^{\prime}} e^{2 \pi i\left(\alpha-\alpha^{\prime}\right) \mu} e^{\frac{i \pi}{2} \mu(Q-\mu)} \frac{\widetilde{S}\left(2 \alpha^{\prime}+\mu \mid b\right)}{\widetilde{S}\left(2 \alpha^{\prime} \mid b\right)} \sum_{n=0}^{\infty} e^{4 \pi i n b \alpha} \mathcal{M}^{n}\left(\alpha^{\prime}, \mu\right)
$$

with coefficients $\mathcal{M}^{n}\left(\alpha^{\prime}, \mu\right)$ defined as

$$
\mathcal{M}^{n}\left(\alpha^{\prime}, \mu\right)=\prod_{k=1}^{n} \frac{\sin \pi b\left(2 \alpha^{\prime}+\mu+(k-1) b\right) \sin \pi b(\mu+(k-1) b)}{\sin \pi b\left(2 \alpha^{\prime}+k b\right) \sin \pi k b^{2}}
$$

solves equation (4.26), i.e.

$$
\left(e^{\frac{b}{2} \partial_{\alpha}}-e^{-\frac{b}{2} \partial_{\alpha}}\right) \widetilde{\mathcal{M}}_{\alpha \alpha^{\prime}}(\mu)=2 \sin 2 \pi b \alpha e^{b \partial_{\mu}} \widetilde{\mathcal{M}}_{\alpha \alpha^{\prime}}(\mu)
$$


It is easy to compute the l.h.s.

$$
\begin{aligned}
\left(e^{\frac{b}{2} \partial_{\alpha}}-e^{-\frac{b}{2} \partial_{\alpha}}\right) \widetilde{\mathcal{M}}_{\alpha \alpha^{\prime}}(\mu)=2 i e^{4 \pi i \alpha \alpha^{\prime}} & e^{2 \pi i\left(\alpha-\alpha^{\prime}\right) \mu} e^{\frac{i \pi}{2} \mu(Q-\mu)} \frac{\widetilde{S}\left(2 \alpha^{\prime}+\mu \mid b\right)}{\widetilde{S}\left(2 \alpha^{\prime} \mid b\right)} \\
& \times \sum_{n=0}^{\infty} e^{4 \pi i n b \alpha} \mathcal{M}^{n}\left(\alpha^{\prime}, \mu\right)\left[\sin \pi b\left(2 \alpha^{\prime}+\mu+2 n b\right)\right]
\end{aligned}
$$

Next, with the help of relation (A.12) the r.h.s. can be written as

$$
\begin{aligned}
2 \sin 2 \pi b \alpha e^{b \partial_{\mu}} & \widetilde{\mathcal{M}}_{\alpha \alpha^{\prime}}(\mu)=i e^{-2 \pi i b \alpha^{\prime}}\left(1-e^{2 \pi i b\left(2 \alpha^{\prime}+\mu\right)}\right) e^{\frac{i \pi}{2}} e^{-i \pi b \mu} \\
& \times e^{4 \pi i \alpha \alpha^{\prime}} e^{2 \pi i\left(\alpha-\alpha^{\prime}\right) \mu} e^{\frac{i \pi}{2} \mu(Q-\mu)} \frac{\widetilde{S}\left(2 \alpha^{\prime}+\mu \mid b\right)}{\widetilde{S}\left(2 \alpha^{\prime} \mid b\right)} \\
& \times\left(1-e^{4 \pi i b \alpha}\right) \sum_{n=0}^{\infty} e^{4 \pi i n b \alpha} \mathcal{M}^{n}\left(\alpha^{\prime}, \mu\right) \frac{\sin \pi b\left(2 \alpha^{\prime}+\mu+n b\right) \sin \pi b(\mu+n b)}{\sin \pi b\left(2 \alpha^{\prime}+\mu\right) \sin \pi b \mu}
\end{aligned}
$$

Denote

$$
\mathcal{N}^{n}\left(\alpha^{\prime}, \mu\right)=\mathcal{M}^{n}\left(\alpha^{\prime}, \mu\right) \frac{\sin \pi b\left(2 \alpha^{\prime}+\mu+n b\right) \sin \pi b(\mu+n b)}{\sin \pi b\left(2 \alpha^{\prime}+\mu\right) \sin \pi b \mu}
$$

Then the last line in equation (C.5) is

$$
\begin{aligned}
\left(1-e^{4 \pi i b \alpha}\right) & \sum_{n=0}^{\infty} e^{4 \pi i n b \alpha} \mathcal{N}^{n}\left(\alpha^{\prime}, \mu\right) \\
= & \sum_{n=0}^{\infty} e^{4 \pi i n b \alpha} \mathcal{N}^{n}\left(\alpha^{\prime}, \mu\right)\left[1-\frac{\mathcal{N}^{n-1}\left(\alpha^{\prime}, \mu\right)}{\mathcal{N}^{n}\left(\alpha^{\prime}, \mu\right)}\right] \\
= & \sum_{n=0}^{\infty} e^{4 \pi i n b \alpha} \mathcal{N}^{n}\left(\alpha^{\prime}, \mu\right)\left[1-\frac{\sin \pi b\left(2 \alpha^{\prime}+n b\right) \sin \pi n b^{2}}{\sin \pi b\left(2 \alpha^{\prime}+\mu+n b\right) \sin \pi b(\mu+n b)}\right] \\
= & \sum_{n=0}^{\infty} e^{4 \pi i n b \alpha} \mathcal{M}^{n}\left(\alpha^{\prime}, \mu\right) \frac{\sin \pi b\left(2 \alpha^{\prime}+\mu+n b\right) \sin \pi b(\mu+n b)-\sin \pi b\left(2 \alpha^{\prime}+n b\right) \sin \pi n b^{2}}{\sin \pi b\left(2 \alpha^{\prime}+\mu\right) \sin \pi b \mu} \\
& =\frac{1}{\sin \pi b\left(2 \alpha^{\prime}+\mu\right)} \sum_{n=0}^{\infty} e^{4 \pi i n b \alpha} \mathcal{M}^{n}\left(\alpha^{\prime}, \mu\right)\left[\sin \pi b\left(2 \alpha^{\prime}+\mu+2 n b\right) \sin \pi b \mu\right]
\end{aligned}
$$

where in the last transformation the following trigonometric identity was used

$$
\begin{aligned}
\left.\sin \pi b\left(2 \alpha^{\prime}+\mu+n b\right) \sin \pi b(\mu+n b)-\sin \pi b\left(2 \alpha^{\prime}+n b\right) \sin \pi n b^{2}\right) & \\
& =\sin \pi b\left(2 \alpha^{\prime}+\mu+2 n b\right) \sin \pi b \mu
\end{aligned}
$$

The sum in the last line of equation (C.7) is the same as in equation (C.4). Finally, collecting the prefactors in equations (C.5), (C.7) and noting that

$$
\frac{i e^{-2 \pi i b \alpha^{\prime}}\left(1-e^{2 \pi i b\left(2 \alpha^{\prime}+\mu\right)}\right) e^{\frac{i \pi}{2}} e^{-i \pi b \mu}}{\sin \pi b\left(2 \alpha^{\prime}+\mu\right)}=2 i
$$

we obtain the desired result (C.3). 
Open Access. This article is distributed under the terms of the Creative Commons Attribution License (CC-BY 4.0), which permits any use, distribution and reproduction in any medium, provided the original author(s) and source are credited.

\section{References}

[1] A.A. Belavin, A.M. Polyakov and A.B. Zamolodchikov, Infinite conformal symmetry in two-dimensional quantum field theory, Nucl. Phys. B 241 (1984) 333 [INSPIRE].

[2] J. Teschner, From Liouville theory to the quantum geometry of Riemann surfaces, hep-th/0308031 [INSPIRE].

[3] N. Nemkov, On fusion kernel in Liouville theory, arXiv:1409.3537 [INSPIRE].

[4] E.P. Verlinde, Fusion rules and modular transformations in $2 D$ conformal field theory, Nucl. Phys. B 300 (1988) 360 [INSPIRE].

[5] N. Drukker, J. Gomis, T. Okuda and J. Teschner, Gauge theory loop operators and Liouville theory, JHEP 02 (2010) 057 [arXiv: 0909.1105] [INSPIRE].

[6] L.F. Alday, D. Gaiotto, S. Gukov, Y. Tachikawa and H. Verlinde, Loop and surface operators in $N=2$ gauge theory and Liouville modular geometry, JHEP 01 (2010) 113 [arXiv:0909.0945] [INSPIRE].

[7] D. Galakhov, A. Mironov and A. Morozov, S-duality and modular transformation as a non-perturbative deformation of the ordinary pq-duality, JHEP 06 (2014) 050 [arXiv: 1311.7069] [INSPIRE].

[8] L. Hadasz, Z. Jaskolski and P. Suchanek, Recursive representation of the torus 1-point conformal block, JHEP 01 (2010) 063 [arXiv:0911.2353] [INSPIRE].

[9] B. Ponsot and J. Teschner, Liouville bootstrap via harmonic analysis on a noncompact quantum group, hep-th/9911110 [INSPIRE].

[10] B. Ponsot and J. Teschner, Clebsch-Gordan and Racah-Wigner coefficients for a continuous series of representations of $U(q)(\mathrm{SL}(2, \mathbb{R}))$, Commun. Math. Phys. 224 (2001) 613 [math/0007097] [INSPIRE].

[11] J. Teschner and G.S. Vartanov, Supersymmetric gauge theories, quantization of $\mathcal{M}_{\mathrm{flat}}$ and conformal field theory, Adv. Theor. Math. Phys. 19 (2015) 1 [arXiv:1302.3778] [InSPIRE].

[12] D. Galakhov, A. Mironov and A. Morozov, S-duality as a $\beta$-deformed Fourier transform, JHEP 08 (2012) 067 [arXiv: 1205.4998] [INSPIRE].

[13] M. Billó, M. Frau, L. Gallot, A. Lerda and I. Pesando, Deformed $N=2$ theories, generalized recursion relations and S-duality, JHEP 04 (2013) 039 [arXiv: 1302.0686] [INSPIRE].

[14] M. Billó, M. Frau, L. Gallot, A. Lerda and I. Pesando, Modular anomaly equation, heat kernel and S-duality in $N=2$ theories, JHEP 11 (2013) 123 [arXiv:1307.6648] [INSPIRE].

[15] N. Nemkov, S-duality as Fourier transform for arbitrary $\epsilon_{1}, \epsilon_{2}$, J. Phys. 47 (2014) 105401 [arXiv: 1307.0773] [INSPIRE]. 